\title{
Editorial Note of Farewell and Gratitude to ESTS Reviewers
}

\author{
DANIEL LEE KLEINMAN, ESTS FOUNDING EDITOR
}

\author{
KATIE VANN, ESTS FOUNDING MANAGING EDITOR
}

\begin{abstract}
In response to the desire of the governing council of the Society for Social Studies of Science to have an online open access Society journal, we were asked to build Engaging Science, Technology, and Society. We did so and launched the journal in 2015. Today, in December 2020, we write to say farewell, and to thank the many reviewers who selflessly gave their time, energy, and expertise to support the journal and the efforts of other authors. On the eve of our departure, we delight in the strength of ESTS and the quality of papers that have been published over the six annual volumes realized since 2015. And in this moment in history, when so many incentives exist for scholars to confine their energy to self-serving activities and show indifference to the quality of literature produced, the good will and actions of the reviewers who have enabled the development and growth of ESTS are what we deeply appreciate. To those ESTS reviewers who are shown below, Thank You. And to those of you who read this, when you see those listed you should know, "There is someone who demonstrated a commitment to quality STS scholarship and to the field."
\end{abstract}

Sincerely, ESTS Founding Editors-Daniel Lee Kleinman and Katie Vann

$\begin{array}{llll}\text { Sara Ackerman } & \text { Caragh Brosnan } & \text { Alessandro Delfanti } & \text { Catherine Grandclement } \\ \text { Samer Alatout } & \text { Elizabeth Buchanan } & \text { Ana Delgado } & \text { Sara Grimes } \\ \text { Mathieu Albert } & \text { Michael Burgess } & \text { Lindsey Dillion } & \text { Julia S. Guivant } \\ \text { Barbara Allen } & \text { Ryan Burns } & \text { Jon Downer } & \text { Frode Guribye } \\ \text { Kirstie Ball } & \text { Lawrence Busch } & \text { Greg Downey } & \text { Hugh Gusterson } \\ \text { Andrea Ballestero } & \text { Jo Cain } & \text { Trevor Durbin } & \text { Erica Haimes } \\ \text { Jessica Barnes } & \text { Jane Calvert } & \text { David Edgerton } & \text { Saul Halfon } \\ \text { Christopher Bear } & \text { Nerea Calvillo } & \text { Kristin Echenfelder } & \text { Sarah Hartley } \\ \text { Ruha Benjamin } & \text { Charles Camic } & \text { Noah Feinstein } & \text { Penny Harvey } \\ \text { Beth Popp Berman } & \text { Annamaria Carusi } & \text { Gary Fine } & \text { Gabriel Hecht } \\ \text { Tim Berard } & \text { Laura Centemeri } & \text { Michael Fisch } & \text { Stefan Helmreich } \\ \text { Rachel Best } & \text { Anita Say Chan } & \text { Des Fitzgerald } & \text { Emmanuel Henry } \\ \text { Nicholas Beuret } & \text { Rob Chilles } & \text { Laura Forlano } & \text { Maja Hertoghs } \\ \text { Stuart Blume } & \text { Talia Dan-Cohen } & \text { Mary Frank Fox } & \text { David Hess } \\ \text { Grant Bollmer } & \text { Morgan Currie } & \text { Scott Frickel } & \text { Vaughan Higgins } \\ \text { Cristobal Bonelli } & \text { David Danks } & \text { John Gardner } & \text { Stephen Hilgartner } \\ \text { Marcel Boumans } & \text { Chris Le Dantec } & \text { Tarleton Gillespie } & \text { Steve Hoffman } \\ \text { Geof Bowker } & \text { Christian Dayé } & \text { Eva Giraud } & \text { Mél Hogan } \\ \text { Daniel Breslau } & \text { Jason Delborne } & \text { Leland Glenna } & \text { Kerry Holden }\end{array}$




\author{
Lily Irani \\ Kirk Jalbert \\ Kelly Joyce \\ David Jones \\ Abby Kinchy \\ Hans Klein \\ Vasilis Kostakis \\ Tong Lam \\ Vincent Lagendijk \\ Rebecca Lave \\ Máire Leane \\ Allison Loconto \\ Myles Lennon \\ Les Levidow \\ Karen Levy \\ Javier Lezaun \\ Max Liboiron \\ Debra Mackinnon \\ Theresa MacPhail \\ Kate Maddalenas \\ Martin Mahony \\ Guillaume Marche \\ Noortje Marres \\ Claire Marris \\ Brad Mehlenbacher
}

Mike Michael

Phil Mirowski

Audra Mitchell

Kelly Moore

Jill Morowski

Manny Moss

Rahul Mukherjee

Michelle Murphy

Daniel Neyland

Kate O'Riordan

Aaron Panofsky

Shobita Parthasarathy

Nassim Parvin

Bart Penders

Kimberley Peters

Michael Pettit

Adriana Petryna

Sarah Pfatteicher

Roopali Phadke

Martyn Pickersgill

Martha Poon

Amit Prasad

Matt Ratto

Catherine Rhodes

David Ribes
Sarah de Rijke

Jody Roberts

David Rojas

Harald Rohracher

John Rudolph

Therese Ryley

Jathan Sadowski

Stevienna de Saille

Amiria Salmond

Eric Schatzberg

Pablo Schyfter

Laura Senier

Kelly Shannon

Nick Shapiro

Susan Silbey

Laurel Smith-Doerr

Johan Soderberg

Matt Stanley

Holger Straßheim

Banu Subramaniam

Sainath Suryanarayanan

Heather Swanson

Peter Taylor

Samuel Taylor-Alexander

Paul B. Thompson
Chris Till

Shashank Tiwari

Victor Toom

Sezin Topçu

Ilke Turkmendag

David Tyfield

Steve Vallas

Roli Varma

Diane Vaughan

Michael Veale

Lea Velho

Michael Vine

Antonia Walford

Miranda Waggoner

Anna Wesselink

Kristoffer Whitney

Cathering Will

Joan Wolf

Caitlin Wylie

Sally Wyatt

Jerry Zee

Thomas Zeller

Malte Ziewitz

Frank de Zwart 